Review

\title{
Emerging trends in COVID-19 treatment: learning from inflammatory conditions associated with cellular therapies
}

\author{
Maria Cancio, $\mathrm{MD}^{1, *}$, Rachele Ciccocioppo, $\mathrm{MD}^{2}$, Patricia R.M. Rocco, MD, PhD ${ }^{3}$, \\ Bruce L. Levine, $\mathrm{MD}^{4}$, Vincenzo Bronte, $\mathrm{MD}^{2}$, Catherine M. Bollard, $\mathrm{MD}^{5}$, \\ Daniel Weiss, MD, $\mathrm{PhD}^{6}$, Jaap Jan Boelens, $\mathrm{MD}, \mathrm{PhD}^{1}$, Patrick J. Hanley, $\mathrm{PhD}^{5}$ \\ ${ }^{1}$ Memorial Sloan Kettering Cancer Center, New York, New York, USA \\ ${ }^{2}$ Department of Medicine, A.O.U.I Policlinico G.B Rossi and University of Verona, Verona, Italy \\ ${ }^{3}$ Laboratory of Pulmonary Investigation, Carlos Chagas Filho Institute of Biophysics, Federal university of Rio de Janeiro, Rio de Janeiro, Brazil \\ ${ }^{4}$ Center for Cellular Immunotherapies and Department of Pathology and Laboratory Medicine, Perelman School of Medicine, University of Pennsylvania, \\ Philadelphia, Pennsylvania, USA \\ ${ }^{5}$ Center for Cancer and Immunology Research, Center for Cancer and Blood Disorders, Children's National Hospital and the George Washington University Cancer \\ Center, George Washington University, Washington, DC, USA \\ ${ }^{6}$ University of Vermont Medical Center, Burlington, Vermont, USA
}

\section{A R T I C L E I N F O}

\section{Key Words:}

cellular therapy

COVID-19

CRS

cytokine release

HLH

hyperimmune response

immunotherapy

IRIS

SARS-CoV-2

\begin{abstract}
A B S T R A C T
Coronavirus disease 2019 (SARS-CoV2) is an active global health threat for which treatments are desperately being sought. Even though most people infected experience mild to moderate respiratory symptoms and recover with supportive care, certain vulnerable hosts develop severe clinical deterioration. While several drugs are currently being investigated in clinical trials, there are currently no approved treatments or vaccines for COVID-19 and hence there is an unmet need to explore additional therapeutic options. At least three inflammatory disorders or syndromes associated with immune dysfunction have been described in the context of cellular therapy. Specifically, Cytokine Release Syndrome (CRS), Immune Reconstitution Inflammatory Syndrome (IRIS), and Secondary Hemophagocytic Lymphohistiocytosis (sHLH) all have clinical and laboratory characteristics in common with COVID19 and associated therapies that could be worth testing in the context of clinical trials. Here we discuss these diseases, their management, and potential applications of these treatment in the context of COVID-19. We also discuss current cellular therapies that are being evaluated for the treatment of COVID-19 and/or its associated symptoms.
\end{abstract}

(c) 2020 International Society for Cell and Gene Therapy. Published by Elsevier Inc. All rights reserved.
Coronavirus disease 2019 (COVID-19) is an infectious illness caused by a novel betacoronavirus strain first recognized in December 2019. The etiological agent of COVID-19, SARS-CoV2, is structurally related to viruses responsible for severe acute respiratory syndrome (SARS$\mathrm{CoV}$ ) and Middle East respiratory syndrome coronavirus (MERS-CoV). These coronavirus strains have been responsible for epidemics and high fatality rates in the past two decades. COVID-19 is an active global health threat with conflicting fatality rate reports, but it is known to be higher in hosts with preexisting medical conditions and/ or advanced age [1].

At the time of this writing, COVID-19 pandemic continues unabated in many parts of the globe. Although most people infected with SARS-CoV2 experience mild to moderate respiratory symptoms and recover with supportive care, certain hosts (even including younger, previously healthy ones) are more likely to develop a precipitous

\footnotetext{
* Correspondence: Maria Cancio, MD, 1275 York Ave, New York, NY 10065, USA.

E-mail address: Canciom@mskcc.org (M. Cancio).
}

clinical decline. Current data support that hyperimmune host reactions triggered by the virus are at least partially responsible for these poor outcomes [2]. Early identification and intervention in these high-risk patients may provide a means to disease amelioration and potentially expedited recovery, although to date no pharmacological therapy has been identified.

A full-bodied worldwide research effort is currently underway to repurpose existing drugs and develop new therapies able to halt progressive disease that leads to respiratory failure and death. Here, we explore how we can apply our extensive knowledge of immune modulation that has been gained from the cellular therapy field to overcome the therapeutic challenges of treating COVID-19. Biological therapies to dampen hyperimmune host responses could potentially be implemented along with supportive care (supplementary oxygen and mechanical ventilatory support when indicated) or in combination with other pharmacotherapies such as antimalarials, antivirals, anti-COVID-19 antibody infusions (convalescent serum), anticoagulation and immunization. 


\section{COVID-19 pathophysiology}

SARS-CoV2 is a betacoronavirus closely related to SARS-CoV. Both viruses use the angiotensin-converting enzyme-related carboxypeptidase (ACE2) receptor to gain entry to cells. This receptor is widely expressed in cardiopulmonary tissues and also in some hematopoietic cells, including monocytes and macrophages [3]. Direct diffuse alveolar damage due to viral targeting of the human respiratory tract epithelium via ACE2 receptors may lead to a hyperimmune response in the host, which can result in the development of acute respiratory failure and ultimately, in some cases, death. Decade-long structural studies on SARS-CoV postulate that viral receptor recognition is one of the most important determinants of its cross-species and human-to-human transmissions, strongly suggesting that SARS-CoV2 uses ACE2 as its receptor [4]. Recently, a cohort of 21 patients with confirmed COVID-19 infection and severe disease showed markedly elevated blood levels of cytokines and chemokines that may predict disease severity [5]. This virus-induced exaggerated host immune response is a previously described phenomenon thought to be responsible for the high fatality rate observed in healthy young adults during the 1918 influenza pandemic and also described with SARS and MERS infections [6].

Early clinical reports of malaise, high fever, chills, anosmia, dysgeusia and hypoxia combined with biochemical markers associated with inflammation and hypercoagulable state seem to be prevalent. Laboratory anomalies such as lymphopenia, high ferritin, elevated lactate dehydrogenase, interleukin (IL)-6, high C-reactive protein and soluble CD25 suggest that SARS-CoV-2 infection may trigger cytokine storm syndrome. This hyper-reactive cytokine release may represent one of the most important negative prognostic factors in patients infected with COVID-19. Interestingly, these responses resemble inflammatory conditions not infrequently seen after hematopoietic stem cell transplant (HCT) and cellular therapies: cytokine release syndrome (CRS), immune reconstitution inflammatory syndrome (IRIS), and secondary hemophagocytic lymphohistiocytosis (sHLH).

\section{Inflammatory conditions associated with cellular therapies}

Three prominent immune dysregulation syndromes that may arise after cellular therapy and stem cell transplantation include CRS, sHLH and IRIS. All three have clinical and laboratory characteristics in common and with patterns described in patients with severe COVID19 (Table 1). These immune responses may be fatal if not recognized and treated promptly.

CRS is a potentially life-threatening, non-antigen-specific toxicity that has been described following use of bispecific antibodies such as blinatumomab, chimeric antigen receptor (CAR)-T cell therapy and other immune therapies including but not limited to anti-thymocyte globulin (ATG) rituximab, alemtuzumab and nivolumab. CRS is one of the two most common treatment-related adverse events observed after CAR-T cell therapy; as such, identification, grading and treatment of this complication has been well described in recent years $[7,8]$. To harmonize the definitions and grading systems for CRS and neurotoxicity, consensus recommendations were recently implemented by the American Society for Transplantation and Cellular
Therapy [9] (Table 3). The clinical manifestations of CRS have variable timing and can range from mild to life-threatening. The onset of CRS may be within hours after therapeutic antibody therapy and resolve within days. In contrast, the typical course of CRS after CAR-T infusion, is 1 to 14 days (median, 2-3 days), and resolution is expected within a few days to weeks. CRS manifests with fever, tachycardia, hypotension, respiratory distress or hypoxemia and is associated with elevated circulating levels of several cytokines including IL-6 and interferon- $\gamma$ [9]. In general, the degree of cytokine activation correlates with the severity of the clinical picture. Other laboratory findings are variable and reflect systemic inflammation. sHLH/ macrophage activation syndrome (MAS) is a syndrome of excessive inflammation and tissue destruction with overlapping features of CRS. In adults, sHLH is most commonly triggered by viral infections. sHLH is characterized by T-cell-mediated inflammation, fulminant cytokine storm, ferritin elevation and multi-organ failure. The HLH diagnostic criteria is based on the HLH Study Group of the Histiocyte Society 2004 description of the combination of five of the following eight characteristics (Table 2): fever, splenomegaly, cytopenias, hypertriglyceridemia, hypofibrinogenemia, high ferritin, hemophagocytosis, low/absent NK-cell activity and high soluble IL-2 receptor. CRS and sHLH/MAS have many features in common, as each reflects the activation of the reticuloendothelial system initiated by T-cellmediated inflammation. In the CAR-T literature, most patients with moderate to severe CRS meet criteria for HLH/MAS with or without organomegaly, lymphadenopathy or evident hemophagocytosis. Patients may meet some of the criteria for HLH/MAS after CAR-T-cell infusion, but this may also reflect CRS [9]. Importantly, the clinical and cytokine profile suggestive of HLH/MAS resolve with CRS resolution; therefore, primary amelioration of CRS with targeted cytokine biologics may be sufficient without using specific HLH pharmacotherapies [10]. A retrospective study of COVID-19 patients found that elevated serum ferritin and IL-6 correlated with increased mortality [2].

IRIS is another hyperinflammatory syndrome with pathogenesis dependent on $\mathrm{CD}^{+} \mathrm{T}$ cells, proinflammatory cytokines, macrophages and other innate immune cells. IRIS is a phenomenon described in human immunodeficiency virus (HIV) patients with severe lymphopenia and underlying opportunistic infections, occurring during immune recovery upon initiation of antiretrovirals and presenting as

Table 2

HLH diagnostic criteria.

HLH
Fever $>38.5$
Splenomegaly
Cytopenia in at least 2 lineages $\left(\mathrm{Hb}<9 \mathrm{~g} / \mathrm{dL}\right.$, platelets $<100000 / \mathrm{mm}^{3}$, ANC
$\quad<1000 / \mathrm{mm}^{3}$ )
Hypertriglyceridemia ( $>265 \mathrm{mg} / \mathrm{dL}$ ) and/or hypofibrinogenemia $(<150 \mathrm{mg} / \mathrm{dL}$ )
Hemophagocytosis in bone marrow, spleen, lymph node or liver
Low or absent NK activity
Ferritin $>500 \mathrm{ng} / \mathrm{mL}$
Elevated soluble CD25 (soluble IL-2 receptor alpha) 2 SD above age-adjusted
$\quad$ lab specific norms ( $>2400 \mathrm{IU} / \mathrm{mL}$ )

The eight diagnostic criteria according to HLH-2004 trial: at least five of eight criteria must be present to make the diagnosis of $\mathrm{HLH}$

Table 1

Comparison of clinical features of COVID-19 and other hyperimmune response syndromes.

\begin{tabular}{|c|c|c|c|c|c|c|c|c|}
\hline & Fever & Hypoxia & Hypotension & Cytokine storm & Cytopenias & Ferritin & Hypofibrinogenemia & Organomegaly \\
\hline IRIS & + & + & $+1-$ & + & - & $+1-$ & $+1-$ & $+1-$ \\
\hline COVID-19 & + & + & $+1-$ & + & + & $\uparrow$ & + & Unknown/+ \\
\hline
\end{tabular}


worsened clinical manifestations of a preexisting infection or unmasking of a clinically silent infection [11].

Another condition in which antimicrobial treatment may trigger IRIS is Whipple's disease, a chronic infection due to Tropheryma whipplei that develops in immunologically susceptible individuals [12]. Here, the initial reduction in $\mathrm{CD} 4^{+}$cell counts and their reconstitution on treatment is more pronounced in those patients who developed IRIS than in those without IRIS [13]. Moreover, an imbalance between activated $\mathrm{CD}^{+} \mathrm{T}$ cells and regulatory $\mathrm{T}$ cells seem to play a crucial role in triggering the cytokine storm. Notably, $T$. whipplei-specific Thelper 1 reactivity remained suppressed before and after emergence of IRIS, thus indicating that flare-up of pathogen-specific immunoreactivity is not instrumental in the pathogenesis of IRIS. This implies that independent of the causal infectious agent, IRIS may possibly emerge in those carrying a specific immunological status and that the therapeutic approach may be the same. Therefore, efforts should be undertaken to identify the predisposing dysfunctional immune condition.

Multiple pathogens cause IRIS, which can manifest systemically or localize to the lung. The mechanisms involved in its immunopathogenesis are complex and not entirely understood [14]. The harmful effect of immune response to viral triggers on immunosuppressed patients who experience IRIS with immune recovery (followed by worsening various infections) has also been well described in the allogeneic HCT literature [15]. Respiratory viruses have been suggested to play a role in the pathogenesis of alloimmune-mediated lung syndromes [16]. It remains to be ascertained whether treatment of COVID-19 in susceptible immunocompromised hosts may trigger IRIS.

The immune dysregulation seen in CRS and sHLH/MAS has recently been described in some patients with severe COVID-19 and overlaps with IRIS (Figure 1 and Table 1); therefore, applying lessons learned from the cell therapy literature may provide important insights into severity classification and prognostic clues [17].

\section{Biologic therapies and immunotherapy used to dampen hyperimmune responses}

\section{IL-6 inhibitors}

Tocilizumab (Actemra ${ }^{\mathrm{TM}}$ ) is a recombinant humanized monoclonal antibody directed against the IL-6 receptor. Endogenous IL- 6 is induced by inflammatory stimuli and mediates a variety of immunological responses. Inhibition of IL- 6 receptors by tocilizumab leads to a reduction in cytokine and acute phase reactant production.

IL-6 is a pleotropic cytokine with both anti- and pro-inflammatory effects. It is mainly produced by $\mathrm{T}$ cells and macrophages upon a tissue injury but also from skeletal muscle, smooth muscle in blood vessels, adipocytes and, notably, by lung epithelial cells [18]. It could be hypothesized that myalgias, microthrombi formation and higher complications in obese patients could be associated to IL- 6 effects. Moreover, IL- 6 crosses the blood-brain barrier and triggers the increase of body temperature through the release of prostaglandin $E_{2}$ (PGE2).

Tocilizumab is indicated for adult patients with autoimmune disorders such as rheumatoid arthritis and was approved by the U.S. Food and Drug Administration (FDA) in 2017 for severe, life-threatening, CAR-T-cell-induced CRS in adults and children. The optimal dose and schedule of tocilizumab for treatment of CAR-T-cell-induced CRS is not known, but dosing instructions in tocilizumab labeling provide general recommendations for safe dosing. The FDA-recommended dose of tocilizumab in CAR-T induced CRS is $8 \mathrm{mg} / \mathrm{kg}$ intravenously (12 $\mathrm{mg} / \mathrm{k}$ for patients $<30 \mathrm{~kg}$ body weight) alone or in combination with corticosteroids.

In a study of 40 hospitalized symptomatic COVID-19 patients, the median IL-6 was reported $27.1 \mathrm{pg} / \mathrm{mL}$ (range $0-430 \mathrm{pg} / \mathrm{mL}$ ). The risk of respiratory failure for patients with IL-6 levels of $\geq 80 \mathrm{pg} / \mathrm{ml}$ was $92 \%$ and thus 22 times higher compared with patients with lower IL6 levels. After reaching an IL-6 value of $80 \mathrm{pg} / \mathrm{mL}$, the median time to mechanical ventilation was 1.5 days (range $0-4$ days) [19] (Table 4). In contrast, some patients with Grade 4 CRS after CART-19 infusion, may have IL-6 levels greater than $600 \mathrm{pg} / \mathrm{mL}$ (120-fold increased from baseline) [20].

The rapid clinical stabilization frequently seen after systemic tocilizumab administration to CRS patients strongly implicates a cytokine array, including IL-8, IL-20, IL-21, IL-22 and dominated by IL-6, in the pathophysiology of the syndrome [9]. The median time from the onset of CRS to the first dose of tocilizumab has been reported to be 4 days (range, $0-18$ days) [21]. Temperature often normalizes within a few hours after tocilizumab administration, whereas the other components of CRS take longer to resolve. In previous CAR-T clinical trials, patients were considered responders if CRS resolved within 14 days of the first dose of tocilizumab, if not more than 2 doses of tocilizumab were needed, and if no drugs other than tocilizumab and corticosteroids were used for treatment. Approximately $70 \%$ of patients achieve a response under this definition [21]. Tocilizumab has a half-life 11-14 days; therefore, if given early, it could dampen

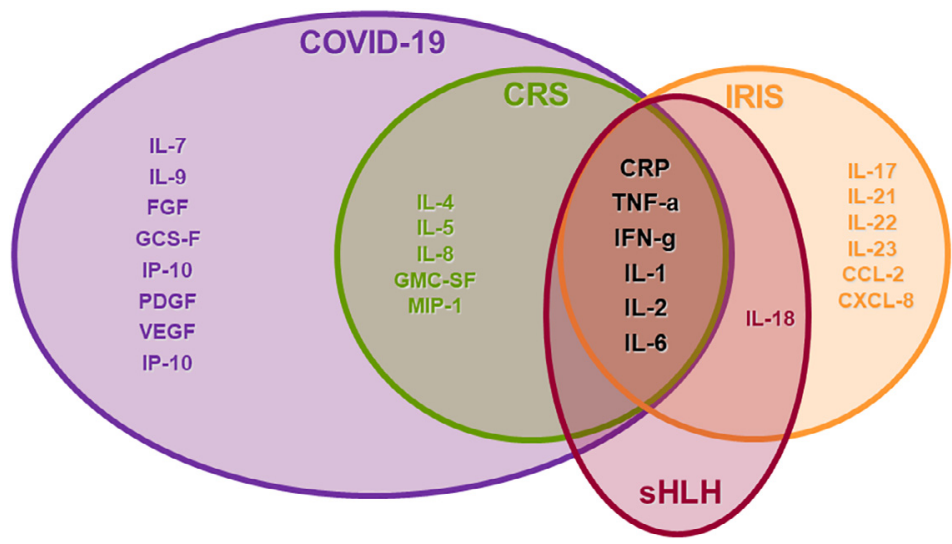

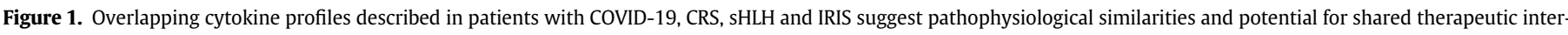

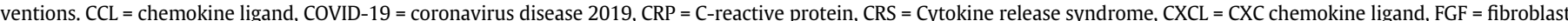

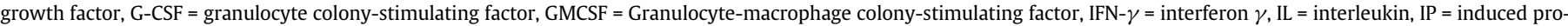

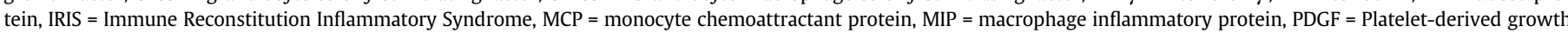

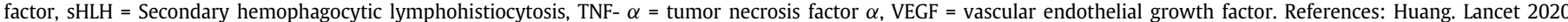
(COVID-19); Brudno. Blood 2019 (CAR-T); Gopal. Eur Resp Review 2017 (IRIS); Schulert. Best Practice Rheum 2014 (sHLH). (Color version of figure is available online). 
hyperimmune responses thought to be related to clinical decline in COVID-19 patients who progress to respiratory failure.

Given the similarities between CRS and COVID-19 hypercytokinemia and clinical symptoms, the clinical benefits and safety of tocilizumab is currently being systematically studied.

The efficacy of IL-6-IL-6R antagonists for the treatment of CRS as well as SHLH underscores the central role of IL- 6 signaling in the pathophysiology of cytokine-driven hyperinflammatory syndromes [22]. Severe COVID-19 cases may benefit from IL-6 pathway inhibition given the associated CRS- and sHLH-like serum cytokine elevations [3].

Currently, tocilizumab is being investigated in an FDA-approved randomized, double-blind, placebo-controlled phase III clinical trial to evaluate its safety and efficacy when used with standard of care in hospitalized adult patients with severe COVID-19 and in a phase II study in Italy approved by the Italian Agency of Pharmaceutics.

Siltuximab (Sylvant ${ }^{\mathrm{TM}}$ ) is a human murine chimeric monoclonal antibody that binds IL- 6 directly, in contrast to tocilizumab that binds to the IL-6 receptor. Siltuximab has a higher affinity for IL-6 than tocilizumab has for the IL-6R making it an attractive consideration in managing CRS. There is some concern that circulating IL-6 levels increase after administration of tocilizumab, contributing to an increased incidence of neurotoxicity $[20,23]$. This does not seem to be a concern with siltuximab, which is the rationale for its proposed benefit in tocilizumab-refractory cases, although no data are currently available on its efficacy. Siltuximab has not been sufficiently studied as a treatment for CRS and its use remains investigational; therefore, it should be considered only as second line agent in cases of COVID-19.

\section{IL-1 inhibitor}

Data from a phase 3 randomized controlled trial of anakinra (Kineret $^{\mathrm{TM}}$ ) in sepsis, showed significant increase in survival in patients with hyperinflammation, without increased adverse events [24]. Currently, Swedish Orphan Biovitrum has an open-label, multicenter clinical trial evaluating the use of anakinra in combination with emapalumab at reducing hyperinflammation in severe COVID-19 patients. Patients in the anakinra arm, will receive anakinra intravenous (IV) infusion four times daily for 15 days ( $400 \mathrm{mg} / \mathrm{day}$, divided in four daily doses).

It is important to note that IL-1 can be detected in the sera of mouse models of cytokine storm; however, correlation with the serum levels of IL-1 and disease severity has not been described for COVID-19 patients. The sensitivity and sensibility of currently available ELISA kits for human IL-1 are being validated. Gene expression and single-cell RNAseq data suggest that a signature related to NF- $\kappa$ B pathway and possibly inflammasome activation might be present [25].

\section{JAK-STAT inhibitors}

Targeting inflammatory cytokine signaling via Janus kinase/signal transducers and activators of transcription (JAK-STAT) inhibition to treat CRS is being reported [26]. Baricitinib, fedratinib and ruxolitinib are potent and selective JAK inhibitors approved for indications such as rheumatoid arthritis and myelofibrosis. All three are powerful anti-inflammatories that, as JAK-STAT signaling inhibitors, are likely to be effective against the consequences of the elevated levels of cytokines (including interferon- $\gamma$ ) typically observed in people with COVID-19 [5].

\section{BTK-inhibitors}

Clinical trials examining the potential benefit for Bruton's tyrosine kinase (BTK) inhibitors such as ibrutinib (Imbruvica ${ }^{\mathrm{TM}}$ ) to protect against lung pathology in patients with COVID-19 are being initiated. The clinical course of six patients who were receiving the drug for Waldenstrom's macroglobulinemia and became ill with COVID-19 was recently reported. The authors proposed that BTK-inhibition may provide protection against lung injury and even improve pulmonary function in hypoxic patients with COVID-19 [27].

\section{Convalescent Plasma}

Immunotherapy with neutralizing antibodies present in convalescent plasma proved to be safe and during the SARS, MERS and 2009 H1N1 influenza epidemics [28,29]. The feasibility of convalescent plasma transfusion to rescue severely ill patients with COVID19 was explored in 10 patients in Wuhan, China. One dose $(200 \mathrm{~mL})$ of convalescent plasma was well tolerated, seemed to significantly improve clinical symptoms within 3 days and resulted in high-level neutralizing antibodies, leading to disappearance of viremia in 7 days. These results should be validated in larger cohorts, preferably with randomized trials [30].

\section{Vaccination}

Currently, there are no approved immunizations for COVID-19. A National Institutes of Health-sponsored phase 1 study is currently evaluating the experimental vaccine mRNA-1273 (Moderna, Inc., Cambridge, Ma, USA) in healthy subjects aged 18 to 55 years. This is a novel lipid nanoparticle-encapsulated mRNA vaccine that encodes for a prefusion stabilized form of the spike (S) protein of SARS-CoV-2. The primary objective of the study is to evaluate the safety and reactogenicity of a 2-dose vaccination schedule of mRNA-1273, given 28 days apart, across three dosages (three different doses being tested, each given twice. E.g. (hypothetically) $50 \mathrm{mg}$ on day $0,50 \mathrm{mg}$ on day $5 ; 100 \mathrm{mg}$ on day $0,100 \mathrm{mg}$ on day $5 ; 500 \mathrm{mg}$ on day $0,500 \mathrm{mg}$ on day 5) (https://www.clinicaltrials.gov/ct2/ show/NCT04283461). Multiple other vaccination studies are currently under development.

\section{Immunomodulation}

\section{MSCS}

MSCs have immunomodulatory properties that make them potential therapeutic tools to repair tissue damage and dampen inflammation in immune-mediated disorders. Indeed, their action is displayed not only in all immune cells, but also in cell populations, such as epithelial cells, endothelial cells, fibroblasts, which take part in inflammatory cascades and are not targeted by other therapies. Moreover, MSCs are an attractive therapeutic option because they elicit no (or a weak) allogeneic immune responses when delivered to a non-identical, non-matched recipient and because they can easily be extracted from a variety of tissues including bone marrow, adipose and placental tissues including umbilical cord blood, and expanded in vitro as well as due to their high safety profile independent to the tissue source and clinical setting [31]. Although they show great promise in the treatment of many immune and inflammatory disorders, the large variability in manufactured cell products, donor cell quality, dosages, formulation as fresh or cryopreserved and differing administration routes have obscured their potential therapeutic benefit. To overcome these hurdles, a careful evaluation of appropriate cell sources, more scientific data and a better mechanistic understanding of immunosuppression of MSCs is needed [32]. The START clinical phase II study has the highest number of MSCtreated patients suffering from (non-COVID) acute respiratory distress syndrome (ARDS; $\mathrm{n}=40$ ), that is, it showed that a single dose of allogeneic bone marrow-derived MSCs did not cause short- or long-term hemodynamic or respiratory adverse events 
over a 60-day follow-up period. However, no significant improvement in the mortality rate related to ARDS was observed. This may be due to differences in the disease severity within the study populations as well as low MSC viability, ranging from only 35 to $80 \%$ [33].

During the SARS-CoV-2 outbreak in China, several clinical studies were open and are still ongoing, with one already published: a single-center open-label pilot investigation from the YouAn Hospital in Beijing administered BM-derived MSCs to seven patients with COVID-19 pneumonia with differing degrees of severity including one patient with critically severe disease requiring ICU care [27]. The MSCs were given as a single intravenous administration at a dose of $10^{6}$ cells $/ \mathrm{kg}$ body weight in $100 \mathrm{~mL}$ of saline at various times after initial symptomatic presentation. The seven patients were categorized as critically severe $(n=1)$, severe $(n=4)$ and common type $(n=2)$. Three additional patients classified as severe received placebo for comparison. Patients were followed for 14 days after MSC or placebo administration and a range of safety and efficacy endpoints were assessed. No infusion toxicities or severe attributable adverse events were observed and patients, including the one categorized as critically severe, apparently demonstrated clinical improvements within 2 to 4 days after MSC administration However, although detailed clinical information as well as information on levels of circulating inflammatory mediators and of inflammatory cell populations were presented, there is a lack of corresponding information for the other six patients or for the three placebo patients. As such, more information regarding inclusion and exclusion criteria, timing of MSC administration relative to disease onset, comorbidities, the clinical course of each patient and a comprehensive evaluation of inflammatory mediators for both treated and placebo patients are required to better determine the potential efficacy of MSC and their mechanisms of action in this setting [35].

In parallel with studies evaluating the potency of MSC for the treatment COVID-19 in China, there are now several centers globally both in the academic and industry-sponsored settings evaluating MSC for this disease. Results from these studies should be forthcoming soon.

\section{Virus-specific T cells}

Virus-specific T cells (VSTs) have been successfully generated against several viruses including cytomegalovirus (CMV), EpsteinBarr virus, adenovirus,[51-55] human herpes virus (HHV)-6, BK polyomavirus (BK), [56] parainfluenza virus, [65] Zika virus, [57] mycobacteria, [58] norovirus, [59] herpes simplex virus (HSV-1), [60] HIV [61] and others. Of these, T cells targeting CMV, EpsteinBarr virus, adenovirus, HHV6, BK virus, parainfluenza and HIV [62] have been tested in patients, with a strong safety profile and many demonstrating promising clinical responses worthy of further clinical evaluation [63]. To broaden access to these cell therapies, several investigators and companies have extended this technology to off-the-shelf allogeneic $T$ cells, where the patient and product are partially human leukocyte antigen (HLA) matched. This could present an appealing opportunity for SARSCoV2-specific T cells. A bank of SARS-CoV2-specific T cells could be created from a diverse range of donors covering diverse HLA alleles, and a personalized product would be selected for each patient based on the degree of HLA match [64]. This would allow for rapid shipment and treatment of patients, albeit in hospitals with existing cell therapy expertise and resources.

\section{Natural killer cells}

Natural killer (NK) cells are lymphocytes that recognize targets via the absence of ligands such as HLA and a complex array of activating and inhibitory receptors. Unlike T cells, NK cells do not rely on peptides presented in the presence of HLA, which makes NK cells an ideal cell therapy for allogeneic, off-the-shelf use. A role for NK cells in the prevention of relapse after HCT has been postulated previously, and recently, CAR NK cells have shown promise in a study of patients with CD19-positive non-Hodgkin's lymphoma or chronic lymphocytic leukemia. Recently, the FDA granted investigators from the biotechnology company Celularity clearance to test whether cryopreserved, allogeneic NK cells derived from placental hematopoietic stem cells are capable of lysing virus-infected cells and control the resulting immune response. However, aside from clinical data of the NK cell product CYNK-001, there is limited public information about the efficacy of CYNK-001 or other NK cells against COVID-19 [36-38].

\section{Extravascular vesicles}

Extravascular vesicles (EVs) are a heterogeneous group of cellderived membranous structures, including exosomes and microvesicles, which are involved in intercellular communication, thus important for multiple physiological and pathological processes [39]. The broad and increasing interest in EVs has opened the opportunity to use exosomes and microvesicles both as biomarkers to follow the progression of various pathological states, as well as potential therapeutic modalities. In particular, MSC-derived EVs have been shown in a range of preclinical studies, including those of acute lung injury, to be as effective as the parent cells themselves [40]. However, there are several issues including but not limited to source, isolation and purification approaches, manufacturing consistency and potency that are in evolution and yet no clinical investigations in patients with ARDS [41]. As such, it is likely premature to consider use of EVs for COVID-19 respiratory disease.

\section{Corticosteroids}

Evidence in patients with SARS and MERS suggests that administration of corticosteroids did not influence mortality but rather delayed viral clearance [42]. Moreover, current interim guidance from the World Health Organization on clinical management of severe acute respiratory infection when novel coronavirus infection is suspected advises against the use of corticosteroids unless indicated for another reason [43]. However, in hyperinflammatory states, immunosuppression is likely to be beneficial (Mehta. Lancet 2020) and may be worth trying for critically ill COVID-19 patients. Further well-designed clinical trials are urgently needed to evaluate the safety and efficacy of steroid therapy in COVID-19.

\section{Drugs with antiviral activity}

The combination of two antiviral molecules (lopinavir, a human immunodeficiency virus type 1 aspartate protease inhibitor, plus ritonavir, an inhibitor of the cytochrome P450 to increase its plasma halflife) has been tried for COVID-19. However, convincing data are lacking as recently demonstrated, the association of lopinavir-ritonavir has proved unfruitful compared with standard of care in an adult cohort of hospitalized patients with severe SARS-CoV-2 pneumonia [44]. Therefore, further antiviral agents have been proposed.

Hydroxychloroquine and chloroquine are drugs that interfere with digestive vacuole function within sensitive malarial parasites by increasing the $\mathrm{pH}$ and inhibiting lysosomal degradation of hemoglobin; inhibit locomotion of neutrophils and chemotaxis of eosinophils and; impair complement-dependent antigen-antibody reactions. Chloroquine has been used for malaria treatment and chemoprophylaxis and hydroxychloroquine is used for treatment of several autoimmune conditions including rheumatoid arthritis. Both drugs have in vitro activity against coronaviruses, with hydroxychloroquine having relatively higher potency against SARS-CoV-2 [45]. On the basis 
Table 3

American Society for Transplantation and Cellular Therapy CAR-T-related CRS consensus grading.

\begin{tabular}{|c|c|c|c|c|}
\hline CRS parameter & Grade 1 & Grade 2 & Grade 3 & Grade 4 \\
\hline $\begin{array}{l}\text { Fever }^{\mathrm{a}} \\
\text { With }\end{array}$ & Temperature $\geq 38^{\circ} \mathrm{C}$ & Temperature $\geq 38^{\circ} \mathrm{C}$ & Temperature $\geq 38^{\circ} \mathrm{C}$ & Temperature $\geq 38^{\circ} \mathrm{C}$ \\
\hline Hypotension & None & Not requiring vasopressors & $\begin{array}{l}\text { Requiring a vasopressor with or without } \\
\text { vasopressin }\end{array}$ & $\begin{array}{l}\text { Requiring multiple vasopressors (exclud- } \\
\text { ing vasopressin) }\end{array}$ \\
\hline $\begin{array}{l}\text { And/or } \\
\text { Hypoxia }\end{array}$ & None & $\begin{array}{l}\text { Requiring low-flow nasal cannula }{ }^{\mathrm{c}} \text { or } \\
\text { blow-by }\end{array}$ & $\begin{array}{l}\text { Requiring high-flow nasal cannula }{ }^{\mathrm{c}} \text {, face- } \\
\text { mask, nonrebreather mask, or venturi } \\
\text { mask }\end{array}$ & $\begin{array}{l}\text { Requiring positive pressure (e.g., CPAP, } \\
\text { BiPAP, intubation and mechanical } \\
\text { ventilation) }\end{array}$ \\
\hline
\end{tabular}

BiPAP; bilevel positive airway pressure; CPAP, Continuous positive airway pressure.

a Fever is defined as temperature $\geq 38^{\circ} \mathrm{C}$ not attributable to any other cause. In patients who have CRS then receive antipyretic or anticytokine therapy such as tocilizumab or steroids, fever is no longer required to grade subsequent CRS severity. In this case, CRS grading is driven by hypotension and/or hypoxia.

b CRS grade is determined by the more severe event: hypotension or hypoxia not attributable to any other cause. For example, a patient with temperature of $39.5^{\circ} \mathrm{C}$, hypotension requiring one vasopressor, and hypoxia requiring low-flow nasal cannula is classified as grade 3 CRS.

c Low-flow nasal cannula is defined as oxygen delivered at $\leq 6 \mathrm{~L} / \mathrm{min}$. Low flow also includes blow-by oxygen delivery, sometimes used in pediatrics. High-flow nasal cannula is defined as oxygen delivered at $>6 \mathrm{~L} / \mathrm{min}$.

Table 4

COVID-19 Laboratory Parameters (adapted from Herold et al., 2020) [19].

\begin{tabular}{llllll}
\hline Laboratory parameters & \multirow{2}{*}{ Evaluable } & Median (range) & \multicolumn{2}{l}{ Mechanical ventilation } & \multirow{2}{*}{$P$ value } \\
& & & No $(\mathrm{n}=27)$ & Yes $(\mathrm{n}=13)$ & \\
\hline Lymphocyte count $(\%)$ & 36 & $19(4-45)$ & $21(4-45)$ & $15(6-26)$ & 0.050 \\
CRP $(\mathrm{mg} / \mathrm{dl})$ & 40 & $2.8(0-31.5)$ & $1.7(0-31.5)$ & $7.8(1.6-17.1)$ & 0.0019 \\
Bilirubin $(\mathrm{mg} / \mathrm{dl})$ & 36 & $0.5(0.2-1.9)$ & $0.5(0.2-1.2)$ & $0.5(0.4-1.9)$ & 0.93 \\
WBC $(\mathrm{G} / \mathrm{l})$ & 40 & $5.295(2.12-308)$ & $4.75(2.12-12.5)$ & $6.64(4.99-308)$ & 0.0014 \\
LDH $(\mathrm{U} / \mathrm{l})$ & 38 & $292(182-1078)$ & $281(182-619)$ & $346(252-1078)$ & 0.0026 \\
PCT $(\mathrm{mg} / \mathrm{ml})$ & 37 & $0(0-5)$ & $0(0-0.6)$ & $0.1(0-5)$ & 0.011 \\
IL6 $(\mathrm{pg} / \mathrm{ml})$ & 37 & $27.1(0-430)$ & $19.6(0-76.5)$ & $121(19.2-430)$ & 0.000012 \\
Platelet count $(\mathrm{G} / \mathrm{ml})$ & 40 & $165(88-440)$ & $186(88-334)$ & $160(1-440)$ & 0.59 \\
Troponin T $(\mathrm{ng} / \mathrm{ml})$ & 34 & $0(0-0.032)$ & $0(0-0.022)$ & $0(0-0.032)$ & 0.018 \\
Creatinine $(\mathrm{mg} / \mathrm{dl})$ & 40 & $0.9(0.4-2.1)$ & $0.9(0.4-1.3)$ & $1.0(0.9-2.1)$ & 0.00034 \\
D-Dimer & 30 & $0.7(0-2.9)$ & $0.6(0-2.2)$ & $1.1(0.6-2.9)$ & 0.028 \\
Ferritin $(\mathrm{ng} / \mathrm{ml})$ & 27 & $644(64-2153)$ & $606(64-1748)$ & $810(431-2153)$ & 0.16 \\
\hline
\end{tabular}

CRP, C-reactive protein; $\mathrm{LDH}=$ lactate dehydrogenase; PCT = Procalcitonin; WBC, white blood cell count.

of limited in vitro and anecdotal data, chloroquine or hydroxychloroquine administered either with or without azithromycin (a macrolide with anti-inflammatory properties) have been recommended for treatment of hospitalized COVID-19 patients in several countries. However, due to the potential for serious adverse effects, especially when combined with other drugs, caution is advised until peerreviewed data are available.

Remdesivir is an investigational antiviral which blocks viral replication by inhibiting RNA synthesis [46]. It has broad antiviral activity that inhibits viral replication through premature termination of RNA transcription and has in vitro activity against SARS-CoV-2 and in vitro and in vivo activity against related beta coronaviruses [45]. Currently, it can be obtained for IV use in hospitalized patients with COVID-19 through an National Institutes of Health-sponsored double-blind placebo-controlled trial (https://clinicaltrials.gov/ct2/show/ NCT04280705), two phase 3 randomized open-label trials (https:// clinicaltrials.gov/ct2/show/NCT04292899 and https://clinicaltrials. gov/ct2/show/NCT04292730) or on an uncontrolled compassionate use basis. In a cohort of patients hospitalized for severe COVID-19 who were treated with compassionate-use remdesivir, clinical improvement was observed in 36 of 53 patients (68\%). Due to study limitations such as cohort size and single arm design, efficacy will require ongoing randomized, placebo-controlled trials [47].

Other potentially interesting treatments currently being studied include the use of emapalumab (anti-interferon- $\gamma$ monoclonal antibody Sobi-IMMUNO-101 study) and the antiparasitic ivermectin [48].

To date, more than 600 worldwide studies and clinical trials investigating COVID-19 are underway. (Appendix 1: COVID-19 Clinical Trials). Rigorous premarketing evaluation of drugs' safety and effectiveness in randomized, controlled trials remains the primary tool for protecting the public from drugs that are ineffective, unsafe or both [49].

\section{Conclusion}

In conclusion, SARS-CoV-2 is a novel betacoronavirus currently causing a pandemic of unprecedented modern proportions. COVID19 ranges from mild viral illness in most hosts to life-threatening disease in a subset of patients who develop hyperimmune inflammatory responses. Further studies aimed at unraveling the immune response in lung and peripheral blood will aid in understanding the risk of developing ARDS is based on preexisting conditions and not the virus itself.

In the absence of FDA- and European Medicines Agency-approved treatments for COVID-19, management currently relies on supportive care. Ongoing reports of clinical and laboratory features of COVID-19 show significant overlap with hyperinflammatory conditions such as CRS, sHLH and IRIS. Early recognition and treatment of hosts who display the hallmarks of CRS, sHLH and SIRS is of utmost importance to reduce morbidity and mortality. A number of therapeutics approved for other indications as well as investigational agents are currently being evaluated in several hundred clinical trials globally. Experiences derived from therapeutics employed for the treatment of complications secondary to immunotherapies, such as CAR-T, bispecific monoclonal antibodies and HCT, may provide a safe framework for the classification and early intervention of patients who are at the highest risk of dying from COVID-19-related complications. Despite the urgency of the pandemic, extreme caution must be exercised to avoid the use of unproven therapeutics based on 
limited data from poorly conducted clinical trials and/or observational data [34,50]. Well-designed and appropriately conducted randomized controlled trials are therefore essential to find a definite answer.

\section{Funding}

This paper was sponsored by the Immuno-Gene Therapy of the International Society for Cell and Gene Therapy. V.B. is supported by Fondazione Cariverona (ENACT project) and Fondazione TIM. M.C. and J.J.B. acknowledge support of the NIH Cancer Center Support Grant P30 CA008748.

\section{Declaration of Competing Interest}

PJH: Stock, other ownership interests, and serves on the board of directors of Mana Therapeutics. Intellectual property related to virusspecific T cells. BL: Stock and other ownership interests in Tmunity Therapeutics; honoraria: Novartis, Terumo, AstraZeneca; consulting or advisory role: Brammer Bio/ThermoFisher Viral Vector services, Avectas, Immuneel, Ori Biotech and Vycellix. VB: BioNTech AG, patent and research collaboration; Ganymed Pharmaceuticals AG, research collaboration; Xios Therapeutics, Scientific Advisory Board; Codiak BioSciences, scientific advisory board; Incyte Corporation, research collaboration. CMB: Stock, Scientific Advisory Board, other ownership interests in Mana Therapeutics. Stock, Cogen Immune Medicine and Neximmune; stock, board of directors, Cabaletta Bio; stock, Scientific Advisory Board, Catamaran Bio; Intellectual property related to virus-specific $\mathrm{T}$ cells and NK cells. JJB: DMC member/chair (consulting); advanced clinical consulting: Avrobio, Takeda, Omeros, Bluebird Bio, BlueRock, Race Oncology; grants: Sanofi (unrestricted grant for clinical trial). The other authors have no commercial, proprietary, or financial interest in the products or companies described in this article.

\section{Author Contributions}

Conception and design of the study: MC, JJB, PH. Analysis and interpretation of data: MC, PH, JJB, BL, RC, PR, VB, DW, CB. Drafting or revising the manuscript: $\mathrm{MC}, \mathrm{PH}, \mathrm{JJB}, \mathrm{BL}, \mathrm{RC}, \mathrm{PR}, \mathrm{VB}, \mathrm{DW}, \mathrm{CB}$. All authors have approved the final article.

\section{Acknowledgments}

The authors thank the Immuno-Gene Therapy Committee of the International Society for Cell and Gene Therapy (ISCT) for sponsoring this article. We also recognize Dr. Alexey Bersenev for providing the open-access COVID-19 clinical trial database.

\section{References}

[1] National Institute of Allergy and Infectious Disease. COVID-19, MERS \& SARS. Updated April 6, 2020. Accessed June 9, 2020. https://www.niaid.nih.gov/diseases-conditions/covid-19.

[2] Channappanavar R, Perlman S. Pathogenic human coronavirus infections: causes and consequences of cytokine storm and immunopathology. Semin Immunopathol 2017;39:529-39.

[3] Moore BJB, June CH. Cytokine release syndrome in severe COVID-19. Science 2020;368:473-4.

[4] Wan S., Fang W., Zheng Y., Li B., Hu Y., et al. Clinical features and treatment of COVID-19 patients in northeast Chongquing [published online ahead of print March 21, 2020]. J Med Virol. https://doi.org/10.1002/jmv.25783.

[5] Huang C, Wang Y, Li X, Ren L, Zhao J, Hu Y, et al. Clinical features of patients infected with 2019 novel coronavirus in Wuhan, China. Lancet 2020:395497. $-506$.

[6] Osterholm MT. Preparing for the next pandemic. N Engl J Med 2005;352:1839-42.

[7] Park JH, Riviere I, Gonen M, Wang X, Senechal B, Curran KJ, et al. Long-term follow-up of CD19 CAR therapy in acute lymphoblastic leukemia. N Engl J Med 2018;378:449-59.
[8] Maude SL, Laetsch TW, Buechner J, Rives S, Boyer M, Bittencourt H, et al. Tisagenlecleucel in children and young adults with B-cell lymphoblastic leukemia. N Engl J Med 2018:378:439-48.

[9] Lee DW, Santomasso BD, Locke FL, Ghobadi A, Turtle CJ, Brudno JN, et al. ASTCT consensus grading for cytokine release syndrome and neurologic toxicity associated with immune effector cells. Biol Blood Marrow Transplant 2019;25:625-38.

[10] Teachey DT, Lacey SF, Shaw PA, Melenhorst JJ, Maude SL, Frey N, et al. Identification of predictive biomarkers for cytokine release syndrome after chimeric antigen receptor T-cell therapy for acute lymphoblastic leukemia. Cancer Discov 2016;6:664-79.

[11] Boulougoura A, Sereti I. HIV infection and immune activation: the role of coinfections. Curr Opin HIV AIDS 2016;11:191-200.

[12] Schneider T, Moos V, Loddenkemper C, Marth T, Fenollar F, Raoult D. Whipple's disease: new aspects of pathogenesis and treatment. Lancet Infect Dis 2008;8:179-90.

[13] Moos V, Feurle GE, Schinnerling K, Geelhaar A, Friebel J, Allers K, et al. Immunopathology of immune reconstitution inflammatory syndrome in Whipple's disease. ] Immunol 2013;190:2354-61.

[14] Gopal R, Rapaka RR, Kolls JK. Immune reconstitution inflammatory syndrome associated with pulmonary pathogens. Eur Respir Rev 2017;26(143):160042.

[15] Versluys AB, Boelens JJ. Morbidity and mortality associated with respiratory virus infections in allogeneic hematopoietic cell transplant: too little defense or harmful immunity? Front Microbiol 2018;9:2795

[16] Versluys B, Bierings M, Luc Murk J, Luc Murk J, Wolfs T, Lindemans C, vd Ent K, et al. Infection with a respiratory virus before hematopoietic cell transplantation is associated with alloimmune-mediated lung syndromes. J Allergy Clin Immunol 2018;141:697-703.

[17] Mehta P, McAuley SF, M. Brown M, Sanchez E, Tattersall RS, Manson JJ. HLH Across Speciality Collaboration, UK. COVID-19: consider cytokine storm syndromes and immunosuppression. Lancet 2020;395:1033-4.

[18] Crestani B, Cornillet P, Dehoux M, Rolland C, Guenounou M, Aubier M. Alveolar type II epithelial cells produce interleukin- 6 in vitro and in vivo. Regulation by alveolar macrophage secretory products. J Clin Invest 1994;94:731-40.

[19] Herold T, Jurinovic V, Arnreich C, Hellmuth JC, von Bergwelt-Baildon M, Klein M, et al. Level of IL-6 predicts respiratory failure in hospitalized symptomatic COVID-19 patients [preprint]. MEDRXIV 2020. https://doi.org/10.1101/ 2020.04.01.20047381.

[20] Chen F, Teachey DT, Pequignot E, Frey N, Porter D, Maude SL, et al. Measuring IL-6 and sIL-6R in serum from patients treated with tocilizumab and/or siltuximab following CAR T cell therapy. J Immunol Methods. 2016;434:1-8.

[21] Le RQ, Li L, Yuan W, Shord SS, Nie L, Habtemariam BA, et al. FDA Approval Summary: Tocilizumab for Treatment of Chimeric Antigen Receptor T Cell-Induced Severe or Life-Threatening Cytokine Release Syndrome. The Oncologist 2018;23:943-7.

[22] Kang S, Tanaka T, Narazaki M, Kishimoto T. Targeting interleukin-6 signaling in clinic. Immunity 2019;50:1007-23.

[23] Nishimoto N, Terao K, Mima T, Nakahara H, Takagi N, Kakehi T. Mechanisms and pathologic significances in increase in serum interleukin-6 (IL-6) and soluble IL-6 receptor after administration of an anti-IL-6 receptor antibody, tocilizumab, in patients with rheumatoid arthritis and Castleman disease. Blood 2008;112:3959-64.

[24] Shakoory B, Carcillo JA, Chatham WW, Amdur RL, Zhao H, Dinarello CA, et at Interleukin-1 receptor blockade is associated with reduced mortality in sepsis patients with features of macrophage activation syndrome: reanalysis of a prior phase III trial. Crit Care Med 2016:44:275-81.

[25] Wen W, Su W, Tang H, Le W, Zhang X, Zheng Y, et al. Immune cell profiling of COVID-19 patients in the recovery stage by single-cell sequencing. Cell Discovery 2020;6:31. https://doi.org/10.1038/s41421-020-0168-9.

[26] Ahmed A, Merrill SA, Alsawah F, Bockenstedt P, Campagnaro E, Devata S, et al. Ruxolitinib in adult patients with secondary haemophagocytic lymphohistiocytosis: an open-label, single-centre, pilot trial. Lancet Haematol 2019;6:e630-7.

27] Treon SP Castillo J, Skarbnik AP, Soumerai LD, Ghobrial IM, Guerrera ML, et al. The BTK-inhibitor ibrutinib may protect against pulmonary injury in COVID-19 infected patients. Blood 2020;135:1912-5

[28] Zhou B, Zhong N, Guan Y. Treatment with convalescent plasma for influenza A (H5N1) infection. N Engl J Med 2007:357:1450-1.

[29] Hung IF, To KK, Lee CK, Lee KL, Chan K, Yan WW, et al. Convalescent plasma treatment reduced mortality in patients with severe pandemic influenza A (H1N1) 2009 virus infection. Clin Infect Dis 2011;52:447-56.

[30] Duan K, Liu B, Li C, Zhang H, Yu T, Qu J, et al. Effectiveness of convalescent plasma therapy in severe COVID-19 patients. Proc Natl Acad Sci U S A 2020;117:9490-6.

[31] Thompson M, Mei SHJ, Wolfe D, Champagne J, Fergusson D, Stewart DJ, et al. Cell therapy with intravascular administration of mesenchymal stromal cells continues to appear safe: an updated systematic review and meta-analysis. EClinicalMedicine 2020;19:100249. https://doi.org/10.1016/j.eclinm.2019.100249.

[32] Gao F, Chiu SM, Motan DA, Zhang Z, Chen L, et al. Mesenchymal stem cells and immunomodulation: current status and future prospects. Cell Death Dis 2016;7: e2062.

[33] Matthay MA, Calfee CS, Zhuo H, Thompson BT, Wilson JG, Levitt JE, et al. Treatment with allogeneic mesenchymal stromal cells for moderate to severe acute respiratory distress syndrome (START study): a randomised phase 2a safety trial. Lancet Respir Med 2019;7:154-62.

[34] Khoury M. Rocco P.R.M. Phinney D.G, Krampera M, Martin I, Viswanathan S. Nolta J.A., et al. Cell-based therapies for COVID-19: proper clinical investigations are essential [published online ahead of print April 27, 2020]. Cytotherapy. https://doi.org/10.1016/j.jcyt.2020.04.089. 
[35] Khoury M., Cuenca J., Cruz F.F., Figueroa F.E., Rocco P.R.M., Weiss D.J.Current status of cell-based therapies for respiratory virus infections [published online ahead of prin April 7, 2020]. Eur Respir J. https://doi.org/10.1183/13993003.00858-2020.

[36] Ruggeri L, Capanni M, Urbani E, Perruccio K, Shlomchik WD, Tosti T, et al. Effectiveness of donor natural killer cell alloreactivity in mismatched hematopoietic transplants. Science 2002;295:2097-100.

[37] Rezvani K, Marin D. Allogeneic BK virus-specific T cells for PML. N Engl J Med 2019;380:106.

[38] Slater H. FDA accepts IND for NK cell therapy CYNK-001 to treat patients with COVID-19. Cancer Network April 3, 2020. https://www.cancernetwork.com/ immuno-oncology/fda-accepts-ind-nk-cell-therapy-cynk-001-treat-patientscovid-19 accessed 23.5.2020.

[39] Lo Cicero A, Stahl PD, Raposo G. Extracellular vesicles shuffling intercellular messages: for good or for bad. Curr Opin Cell Biol 2015;35:69-77.

[40] Shah TG, Predescu D, Predescu S. Mesenchymal stem cells-derived extracellular vesicles in acute respiratory distress syndrome: a review of current literature and potential future treatment options. Clin Transl Med 2019;8:25.

[41] Thery C, Witwer KW, Aikawa E, Alcaraz MJ, Anderson JD, Andriantsitohaina R, et al. Minimal information for studies of extracellular vesicles 2018 (MISEV2018): a position statement of the International Society for Extracellular Vesicles and update of the MISEV2014 guidelines. J Extracell Vesicles 2018;7:1535750.

[42] Russell CD, Millar JE, Baillie JK. Clinical evidence does not support corticosteroid treatment for 2019-nCoV lung injury. Lancet 2020;395:473-5.

[43] World Health Organization. Clinical management of severe acute respiratory infection when novel coronavirus (nCoV) infection is suspected. 27 May 2020. https://www who.int/publications-detail/clinical-management-of-severe-acute-respiratory-infection-when-novel-coronavirus-(ncov)-infection-is-suspected

[44] Cao B, Wang Y, Wen D, Liu W, Wang J, Fan G, et al. A trial of lopinavir-ritonavir in adults hospitalized with severe COVID-19. N Engl J Med 2020;382:1787-99.

[45] Wang M, Cao R, Zhang L, Yang X, Liu J, Xu M, et al. Remdesivir and chloroquine effectively inhibit the recently emerged novel coronavirus (2019-nCoV) in vitro. Cell Res 2020;30:269-71

[46] Agostini M.L., Andres E.L., Sims, Graham R.L., Sheahan T.P., Lu X., et al. Coronavirus susceptibility to the antiviral remdesivir (GS-5734) is mediated by the viralpolymerase and the proofreading exoribonuclease. mBio 2018;9 https://dx.doi.org/ 10.1128/mBio.00221-18.

[47] Grein J, Ohmagari N, Shin D, Diaz G, Asperges E, Castagna A. Compassionate use of remdesivir for patients with severe Covid-19. N Engl J Med 2020;382:2327-36. https://doi.org/10.1056/NEJMoa2007016.

[48] Caly L, Druce JD, Catton MG, Jans DA, Wagstaff KM. The FDA-approved drug ivermectin inhibits the replication of SARS-CoV-2 in vitro. Antiviral Res 2020;178:104787.

[49] Rome B.N., Avorn J.Drug evaluation during the Covid-19 pandemic [published online ahead of print April 14, 2020]. N Engl J Med.

[50] Goodman JL, Borio L. Finding effective treatments for COVID-19: scientific integrity and public confidence in a time of crisis. JAMA Network 2020. https://jamanetwork.com/journals/jama/fullarticle/2764823 Accessed 23.5.2020.

[51] Walter EA, Greenberg PD, Gilbert MJ, Finch RJ, Watanabe KS, Thomas ED, et al. Reconstitution of cellular immunity against cytomegalovirus in recipients of allo- geneic bone marrow by transfer of T-cell clones from the donor. N Engl J Med 1995;333(16):1038-44

[52] Leen AM, Myers GD, Sili U, Huls MH, Weiss H, Leung KS, et al. Monoculturederived T lymphocytes specific for multiple viruses expand and produce clinically relevant effects in immunocompromised individuals. Nat Med 2006;12 (10):1160-6.

[53] Hanley PJ, Melenhorst JJ, Nikiforow S, Scheinberg P, Blaney JW, Demmler-Harrison $\mathrm{G}$, et al. CMV-specific $\mathrm{T}$ cells generated from naive $\mathrm{T}$ cells recognize atypical epitopes and may be protective in vivo. Sci Transl Med 2015;7(285):285ra63.

[54] Hanley PJ, Shaffer DR, Cruz CR, Ku S, Tzou B, Liu H, et al. Expansion of T cells targeting multiple antigens of cytomegalovirus, Epstein-Barr virus and adenovirus to provide broad antiviral specificity after stem cell transplantation. Cytotherapy 2011:13(8):976-86

[55] Hanley PJ, Cruz CR, Savoldo B, Leen AM, Stanojevic M, Khalil M, et al. Functionally active virus-specific $\mathrm{T}$ cells that target CMV, adenovirus, and EBV can be expanded from naive T-cell populations in cord blood and will target a range of viral epitopes. Blood 2009;114(9):1958-67.

[56] Gerdemann U, Keirnan JM, Katari UL, Yanagisawa R, Christin AS, Huye LE, et al. Rapidly generated multivirus-specific cytotoxic T lymphocytes for the prophylaxis and treatment of viral infections. Mol Ther 2012;20(8):1622-32.

[57] Hanajiri R, Sani GM, Hanley PJ, Silveira CG, Kallas EG, Keller MD, et al. Generation of Zika virus-specific T cells from seropositive and virus-naive donors for potential use as an autologous or "off-the-shelf" immunotherapeutic. Cytotherapy 2019;21(8):840-55.

[58] Patel S, Lang H, Sani G, Freeman AF, Leiding J, Hanley PJ, et al. Mycobacteria-Specific T Cells May Be Expanded From Healthy Donors and Are Near Absent in Primary Immunodeficiency Disorders. Front Immunol 2019;10:621.

[59] Hanajiri R, Sani GM, Saunders D, Hanley PJ, Chopra A, Mallal SA, et al. Generation of Norovirus-Specific T Cells From Human Donors With Extensive Cross-Reactivity to Variant Sequences: Implications for Immunotherapy. J Infect Dis 2020;221 (4):578-88

[60] Ma CK, Clancy L, Deo S, Blyth E, Micklethwaite KP, Gottlieb DJ. Herpes simplex virus type 1 (HSV-1) specific T-cell generation from HLA-A1- and HLA-A2-positive donors for adoptive immunotherapy. Cytotherapy 2017;19(1):107-18.

[61] Patel S, Hanajiri R, Grant M, Saunders D, Van Pelt S, Keller M, et al. HIV-Specific T Cells Can Be Generated against Non-escaped T Cell Epitopes with a GMP-Compliant Manufacturing Platform. Mol Ther Methods Clin Dev 2020;16:11-20.

[62] Sung JA, Patel S, Clohosey ML, Roesch L, Tripic T, Kuruc JD, et al. Margolis, HIVSpecific, Ex Vivo Expanded T Cell Therapy: Feasibility, Safety, and Efficacy in ARTSuppressed HIV-Infected Individuals. Mol Ther 2018;26(10):2496-506.

[63] Fatic A, Zhang N, Keller MD, Hanley PJ. The pipeline of antiviral T-cell therapy: what's in the clinic and undergoing development. Transfusion 2020;60(1):7-10.

[64] Withers B, Clancy L, Burgess J, Simms R, Brown R, Micklethwaite K, et al. Establishment and Operation of a Third-Party Virus-Specific T Cell Bank within an Allogeneic Stem Cell Transplant Program. Biol Blood Marrow Transplant 2018;24 (12):2433-42

[65] McLaughlin LP, Lang H, Williams E, Wright KE, Powell A, Cruz CR, et al. Human parainfluenza virus-3 can be targeted by rapidly ex vivo expanded T lymphocytes. Cytotherapy 2016;18(12):1515-24. 